\title{
Targeting MPS1 Enhances Radiosensitization of Human Glioblastoma by Modulating DNA Repair Proteins
}

\author{
Uday B. Maachani ${ }^{1}$, Tamalee Kramp ${ }^{1}$, Ryan Hanson ${ }^{1}$, Shuping Zhao ${ }^{1}$, Orieta Celiku ${ }^{1}$, Uma \\ Shankavaram ${ }^{1}$, Riccardo Colombo ${ }^{2}$, Natasha J. Caplen ${ }^{3}$, Kevin Camphausen ${ }^{1}$, and Anita \\ Tandle $^{1}$
}

${ }^{1}$ Radiation Oncology Branch, National Institutes of Health, Bethesda; Maryland ${ }^{2}$ Nerviano Medical Sciences srl, Oncology, Nerviano, Italy ${ }^{3}$ Genetics Branch, National Institutes of Health, Bethesda; Maryland

\begin{abstract}
To ensure faithful chromosome segregation, cells use the spindle assembly checkpoint (SAC), which can be activated in aneuploid cancer cells. Targeting the components of SAC machinery required for the growth of aneuploid cells may offer a cancer cell specific therapeutic approach. In this study, the effects of inhibiting Monopolar spindle 1, MPS1 (TTK), an essential SAC kinase, on the radiosensitization of glioblastoma (GBM) cells was analyzed. Clonogenic survival was used to determine the effects of the MPS1 inhibitor, NMS-P715 on radiosensitivity in multiple model systems including: GBM cell lines, a normal astrocyte and a normal fibroblast cell line. DNA double strand breaks (DSBs) were evaluated using $\gamma \mathrm{H} 2 \mathrm{AX}$ foci and cell death was measured by mitotic catastrophe evaluation. Transcriptome analysis was performed via unbiased microarray expression profiling. Tumor xenografts grown from GBM cells were used in tumor growth delay studies. Inhibition of MPS1 activity resulted in reduced GBM cell proliferation. Further, NMSP715 enhanced the radiosensitivity of GBM cells by decreased repair of DSBs and induction of post-radiation mitotic catastrophe. MNS-P715 in combination with fractionated doses of radiation significantly enhanced the tumor growth delay. Molecular profiling of MPS1 silenced GBM cells showed an altered expression of transcripts associated with DNA damage, repair and replication including the DNA-dependent protein kinase (PRKDC/DNAPK). Next, inhibition of MPS1 blocked two important DNA repair pathways. In conclusion, these results not only highlight a role for MPS1 kinase in DNA repair and as prognostic marker but also indicate it as a viable option in glioblastoma therapy.
\end{abstract}

\section{Keywords}

Glioblastoma multiforme; MPS1; DNAPK; DNA damage; DNA repair; radiation

*Corresponding Author: Anita Tandle, Ph.D., Radiation Oncology Branch, National Cancer Institute, Building 10, Room B3B100, Bethesda, MD 20892, Telephone: 301-443-4402, Fax Number: 301-480-1434, tandlea@ mail.nih.gov. 


\section{Introduction}

Aneuploidy is a hallmark of cancer cells in which chromosomes are inappropriately partitioned between daughter cells due to aberrant mitosis. Faithful segregation of chromosomes during each cell division is normally ensured by the mitotic spindle assembly checkpoint (SAC) $(1,2)$. It is a highly robust response and alterations in this pathway are frequently observed in a variety of human cancers (3). It has long been thought that mutation of the genes that control chromosome segregation during mitosis may explain the high rate of chromosomal instability and aneuploidy, which is characteristic of most solid tumors, including glioblastomas (GBMs) $(4,5)$. Monopolar spindle 1 (MPS1) an essential SAC kinase, was originally identified as a protein kinase that is overexpressed in human tumor cells and a kinase associated with cell proliferation in mouse embryonic carcinoma cells ( 3 , $4,6,7)$. MPS1 functions in several aspects of cell cycle control, including mitotic spindle assembly checkpoint activation, proper mitotic progression, centrosome duplication, chromosome alignment, error correction of kinetochore-microtubule attachment, and recruitment of SAC components to kinetochores (8-10). Like many cell-cycle regulators, MPS1 transcription is deregulated in a variety of human tumors and elevated MPS1 mRNA levels are found in several human cancers, including thyroid papillary carcinoma, breast cancer, gastric cancer, bronchogenic carcinoma, and lung cancers $(6,11,12)$. Furthermore, high levels of MPS1 correlate with a more aggressive histological grade in breast cancers (13). Several lines of evidence implicate MPS1 in the genotoxic stress response, such as stress caused by DNA damage. Upon exposure to X-ray or UV irradiation, MPS1 causes robust mitotic arrest by direct interaction with CHEK2 and any disruption of the positive feedback loop between these two genes attenuates the DNA damage checkpoint $(14,15)$.

Approximately 50\% of all cancer patients and almost all patients with GBM receive radiotherapy either alone or in combination with other treatment modalities $(16,17)$. Any improvement in the efficacy of radiotherapy will therefore benefit a large number of patients. Further, one of the biological factors that affects radiotherapy outcome is intrinsic radiation damage repair capacity of tumor cells. Modulating the response to ionizing radiation through the inhibition of DNA repair has been a longstanding focus in translational radiotherapy research and represent an attractive target for new therapeutic modalities (17). In the current study, we show that, inhibition of MPS1 abrogates DNA repair following RT allowing an accumulation of DNA damage and as a consequence, cells eventually undergo mitotic catastrophe. Combination of MPS1 inhibition with irradiation increased the radiosensitivity of GBM cells.

\section{Materials and Methods}

\section{Cell lines and drug treatment}

The LN18 (ATCC, (Manassas, VA) and the U251 (National Cancer Institute Frederick Tumor Repository) human GBM cell lines were grown in Dulbecco's Modified Eagle Medium (DMEM) (Invitrogen, Carlsbad, CA) with 10\% fetal bovine serum (FBS), and maintained at $37^{\circ} \mathrm{C}, 5 \% \mathrm{CO}_{2}$. MRC9 (human fetal lung fibroblasts) were obtained from ATCC (CCL-212) and maintained in minimum essential medium supplemented with $10 \%$ FBS, glutamine, sodium pyruvate and non-essential amino acids. Human brain astrocytes 
were purchased from ScienCell (\#1800, Carlsbad, CA) and grown in Astrocyte Medium with the recommended supplements as per manufacturer's instructions and used between passages 3-6. NMS-P715 was obtained from Nerviano Medical Sciences, Nerviano, Italy (18). Drug was reconstituted in dimethyl sulfoxide (DMSO) and stored at $-20^{\circ} \mathrm{C}$. Cells were plated 24 hours prior to drug treatment and treated with NMS-P715 at the concentrations indicated in each experiment.

\section{siRNA-based analysis, gene expression profiling and Ingenuity Pathway Analysis (IPA)}

For siRNA transfections, 2-pmol siMPS1 (siMPS1_2 5'

TTGGACTGTTATACTCTTGAA3', SI00050701; siMPS1_7

5'TCCGACTTATGATTATGAAA3', SI02223214; siMPS1_9

5'CAGAAATAGGTTACCGGAATT 3', SI03062745 (Qiagen Inc., Germantown, MD)

were complexed with RNAi Max lipid transfection reagent (Invitrogen) in DMEM media for 15 minutes at ambient temperature. Two thousand cells suspended in DMEM supplemented with 20\% FBS were then added. Plates were maintained at ambient temperature for 15 minutes before being placed at $37^{\circ} \mathrm{C} / 5 \% \mathrm{CO}_{2}$. Cell viability was assessed five days post siRNA transfection through quantification of ATP (CellTiter-Glo luminescent Reagent, Promega, Madison, WI). Untransfected cells and wells transfected with negative (All star siNegative [siNeg], Qiagen) and positive (All star siCelldeath, Qiagen) control siRNAs were used as controls. RNA and protein for qPCR and western blot analysis were harvested 48 hours post siRNA transfection. To generate a gene expression profile of MPS1 silenced cells, total RNA was extracted (after 6 hour, 24 hour and 48 hour post-transfection) from siMPS1 and siNegative transfected cells and gene expression was assayed using Affymetrix arrays. Analysis was carried out as previously described (19). Data analysis was done with the use of R packages from the Bioconductor project (www.bioconductor.org). The datasets were normalized using MAS5 algorithm. Probesets from each array were mapped to official gene symbols. When multiple probesets were mapped to the same gene, the expressions were processed using customized script to rule out combining values with potential splice variants. In short, a Pearson correlation was computed between all probe sets for a given gene and the two profiles with highest correlation were averaged to represent the gene. Zscore transformation was used to adjust the systematic bias of datasets. An additional filter with standard deviation < 0.3 was implemented to remove invariant genes. Between group siMPS1 (at 6, 24 and 48 hours), differences were compared using a one-way analysis of variance (ANOVA) with posthoc Scheffe analysis. Genes changed between siMPS1 and siNeg conditions ( $\mathrm{p} \leq 0.05$ and fold change $\geq 1.33$ ) at each time point were further analyzed using IPA software (Ingenuity Systems, Mountain View, CA).

\section{Real Time quantitative PCR (qPCR)}

qPCR was carried out using Quantitect Reverse Transcription and SYBR Green PCR kits according to manufacturer's specifications (Sigma-Aldrich, USA). 100-200ng RNA was used for each reverse transcription reaction. Pre-validated primer sets for MPS1, DNAPK/ $P R K C D C$ and TOPO2A were used (Sigma). Reactions were run in an Applied Biosystems 7500 Real Time PCR thermal cycler, and the $2^{-\Delta \Delta C t}$ method was used to calculate relative expression. 


\section{Western blot analysis}

Cell pellets were lysed on ice in RIPA buffer (Pierce, Rockford, IL) supplemented with Complete Mini EDTA-free Protease Inhibitor Cocktail (Roche, Indianapolis, IN) and Phosphatase Inhibitor Cocktail (Sigma, St. Louis, MO). Nuclear cell extracts used in DNA repair assays were prepared using the NE-PER Nuclear Extraction kit (Thermo Scientific, Maine) and did not contain Phosphatase Inhibitor Cocktail. Protein concentrations were determined by Bradford assay (Bio-Rad, Hercules, CA). Protein $(50 \mu \mathrm{g})$ was diluted 1:5 in $5 \mathrm{X}$ protein loading buffer (Fermentas, Glen Burnie, MD), boiled at $80^{\circ} \mathrm{C}$ for 5 minutes, electrophoresed on a 4-20\% Tris-Glycine gel, and transferred using a Trans-Blot Turbo Transfer System (Bio-Rad, Hercules, CA). Membranes were blocked in 5\% Non-fat milk powder (BioRad), incubated with primary antibody overnight at $4^{\circ} \mathrm{C}$, incubated with HRPcoupled secondary antibody 1 hour at room temperature, developed with Visualizer Western Blot Detection Kit (Millipore, Billerica, MA), and visualized on a LAS-4000 imager (Fujifilm, Edison, NJ). Membranes were stripped with Re- Blot Plus Mild (Millipore, Billerica, MA) and re-blocked and probed for additional proteins of interest. The following antibodies were used at 1:1000 dilutions: human anti-MPS1 (05-683, Millipore); mouse anti-actin (MAB 1501R, Millipore), human anti-rabbit DNAPK (04-1024, Millipore), pDNAPK S2056 (ab18192, abcam) and human anti-rabbit TOPO2A (4733, Cell Signaling Technology). Secondary antibodies, goat anti-rabbit-HRP and goat anti-mouse-HRP (Santa Cruz Biotechnology, Santa Cruz, CA) were used at 1:10,000 dilution.

\section{In vitro Non-homologous end joining (NHEJ) activity and Homologous Recombination (HR) assays}

Standard cell free in vitro non-homologous end joining assay (NHEJ) was performed as described previously with some minor modifications (20). Linearized plasmid substrate with cohesive ends was incubated with $1 \mu \mathrm{g}$ of nuclear protein extracts, $1 \mathrm{mM}$ ATP and $1 \mathrm{mM}$ DTT in the end joining reaction buffer at $37^{\circ} \mathrm{C}$ for 1 hour. The reaction mixture was deproteinized with proteinase $\mathrm{K}$ at $65^{\circ} \mathrm{C}$ for $30 \mathrm{~min}$, electrophoresed on a $0.7 \%$ agarose gel and NHEJ products were detected by staining with SybrGreen (Invitrogen). A detailed protocol is given in the Supplementary methods. A PCR based Homologous Recombination assay was performed using Homologous Recombination Assay Kit according to the manufacturer's instructions (Norgen Biotek Corp., Ontario, Canada). A detailed protocol is given in the Supplementary methods.

\section{Clonogenic assay}

Cells were seeded into six-well tissue culture plates and allowed to attach for six hours. NMS-P715 or DMSO control was added to the culture media for two hours. For combination treatment, NMS-P715 or DMSO control was added to the culture media for two hours followed by irradiation with wash after irradiation. Twelve days after seeding, colonies were stained with crystal violet. The number of colonies containing at least 50 cells was determined and the surviving fractions were calculated. For combination treatment, survival curves were generated after normalizing for the cytotoxicity generated by NMSP715 alone. 


\section{Immunofluorescence staining for $\gamma \mathrm{H} 2 \mathrm{AX} / \mathrm{RAD} 51$ foci}

Immunofluorescence staining and counting of $\gamma \mathrm{H} 2 \mathrm{AX}$ nuclear foci was performed as previously described (21). The following antibody was used at 1:1000 dilutions: anti- $\gamma$ H2AX mouse (Millipore, 05-636). Slides were mounted and images captured using Olympus FSX100 fluorescent microscope. For each treatment condition, foci were determined in at least 50 cells. Image-J (NIH) software was used to analyze the mean number of foci with combined area of $\gamma$-H2AX foci per nucleus.

\section{Mitotic catastrophe}

Immunofluorescence staining and counting of mitotic catastrophe foci was performed as previously described (21). Cells were grown in four-well chamber slides, fixed with methanol for 15 minutes at $-20^{\circ} \mathrm{C}$, washed three times with PBS, blocked with $1 \%$ BSA three times for 10 minutes, and stained overnight at $4{ }^{\circ} \mathrm{C}$ with mouse anti-a-tubulin antibody (Sigma) at 1:1000 dilution. Cells were washed three times with 1\% BSA, and were stained with goat anti-mouse-Alexa Fluor 594 (Invitrogen) at 1:200 dilution for two hours at room temperature. Cells were washed three times with $1 \%$ BSA and slides were mounted in Vectashield mounting medium with DAPI (Vector Labs, Burlingame, California). Images were viewed and captured on a fluorescent microscope (Leica, Leica Microsystems, Germany). The presence of giant cells with multi-lobulated nuclei and aberrant mitoses was the criterion for defining cells undergoing mitotic catastrophe. For each treatment condition 150 cells were scored; the average of three separate counts of the same cells is reported.

\section{In vivo tumor growth and irradiation}

All animal studies were conducted in accordance with the principles and procedures outlined in the NIH Guide for the Care and Use of Animals. Four to eight six week old, female, Athymic NCr nu/nu, nude mice, (NCI Animal Production Program, Frederick, MD) were used for all in vivo studies. Animals were caged in groups of 5 or fewer and fed animal chow and water ad libitum. A single cell suspension $\left(5 \times 10^{6}\right)$ of U251 cells was implanted on the lateral aspect of the rear leg. When tumors reached $\approx 140 \mathrm{~mm}^{3}([L \times W 2] / 2)$, animals were randomized into four groups: vehicle ( $0.5 \%$ methyl cellulose) treated controls, NMS-P715 $100 \mathrm{mg} / \mathrm{kg}$, RT, and NMS-P715+RT. Mice were given five doses of NMS-P715 by gavage from Monday through Friday, followed by 4-Gy local irradiation two hours after the fifth dose. The treatment was repeated one more time with a two days gap in between. Irradiation treatment was performed with mice placed in well-ventilated plexiglass jigs with shielding for the entire torso of the mouse along with critical normal structures of the head (e.g., ears, eyes, neck) using a XRad 320 Xirradiator (Precision X-Ray, Inc.) at $320 \mathrm{kV}$ x-ray and a dose rate of $289.8 \mathrm{cGy} / \mathrm{min}$. Sham-irradiated mice served as the control group. To obtain tumor growth curves, perpendicular diameter measurements of each tumor were made twice a week with digital calipers, and volumes were calculated using formula, $\left(\left[L \times W^{2}\right] \times \pi / 2\right)$. Tumors were measured two times per week; animals were euthanized when tumors reached $\geq 1600 \mathrm{~mm}^{3}$. Tumor volumes were interpolated from fit spline curves for all groups the day control tumors had undergone exactly three doublings. Group means \pm standard error is reported. 
For drug kinetic studies, tumor bearing animals were given various doses (either 1 dose, 3 doses or 5 doses $)$ of NMS-P715 (100 mg/kg) 24 hours apart by oral gavage and tumors were harvested 6 hours after the last treatment.

\section{Bioinformatics analysis of publically available datasets}

MPS1 expression levels were correlated to patient survival by curating publically available datasets. A detailed analysis with statistical methods is given in the Supplementary methods.

\section{Statistical analysis}

Data presented are the mean \pm the standard deviation from three independent experiments unless indicated otherwise. All statistical tests were two-sided. For comparisons between groups, a Student's $t$ test was used. All analyses were completed using GraphPad Prism software (GraphPad Prism Inc., San Diego, CA).

\section{Results}

\section{MPS1 inhibition affects cell proliferation in GBM cells}

Previously, we conducted an siRNA-based screen focused on the human kinome in GBM cells and identified the genes required for viability of GBM cells, including the mitosisassociated kinase PLK1 (23). Another mitosis-associated kinase gene this siRNA screen identified as potentially required for GBM cell survival was MPS1. Confirmation of this observation is shown in Supplementary Figure 1. We used 3 non-overlapping siMPS1 siRNAs. A substantial decrease in MPS1 mRNA (Supplementary Figure 1A) and protein (Supplementary Figure 1B) mediated by siRNAs corresponding to MPS1 (siMPS1) was observed with all the three siRNAs. In all the subsequent siMPS1 experiments we used siMPS1_2 siRNA. The decreased expression of MPS1 transcript and subsequent reduction in protein levels was associated with a significant reduction in the viability of the GBM cell line U251 (Figure 1A).

\section{Pharmacologic MPS1 inhibition affects cell proliferation by induction of mitotic catastrophe in GBM cells}

MPS1 encodes the mitotic kinase, Monopolar spindle 1. Recently, an ATP-competitive inhibitor of MSP1, NMS-P715 has been developed (18). To confirm and extend our siRNAbased findings we exposed two GBM tumor cell lines, LN18 and U251, a normal fibroblast line (MRC9) and a normal astrocyte line, to increasing concentrations of NMS-P715. GBM cells LN18 and U251 treated with NMS-P715 showed a statistically significant inhibition of cell proliferation with a concentration-dependent decrease in cell viability of GBM cells over time and a minimal effect on the viability of normal cells (MRC9 and normal astrocytes) (Figure 1B). As a functional assay for MPS1 kinase inhibition, we determined if NMS-P715 treated GBM cells and normal astrocytes exhibited mitotic catastrophe. Cells with nuclear fragmentation, defined as the presence of two or more distinct nuclear lobes within a single cell, were classified as being in mitotic catastrophe. As shown in figure (Figure 2A), inhibition of MPS1 resulted in a significant increase in the percentage of cells undergoing mitotic catastrophe at 24 hours in both LN18 and U251 GBM cells as compared with untreated cells ( $\mathrm{p}=0.00006$ and 0.003 , respectively), indicating one of the mechanisms 
of cell kill is induction of mitotic catastrophe. Consistent with this, normal astrocytes treated with NMS-P715 did not undergo mitotic catastrophe.

\section{MPS1 inhibition enhances the radiosensitivity of GBM cells}

Next, we measured the effect of the MPS1 inhibitor, NMS-P715 on the enhancement of radiosensitivity by clonogenic survival analysis in three different cell lines (Figure 2B). Cells were plated at specific clonogenic density, allowed to attach (six hours) and $1 \mu \mathrm{M}$ NMS-P715 inhibitor (Figure 2B) was added two hours before irradiation. After irradiation, fresh drug-free medium was added, and colonies were stained 12 days later. The survival efficiencies were 35\% and 32\% in LN18 and U251 cells respectively. NMS-P715 treatment resulted in an increase in the radiosensitivity of each of the two GBM cell lines tested. The dose enhancement factors (DEF) at a surviving fraction of 0.1 for LN18 and U251, were 1.40 and 1.32 respectively (Figure $2 \mathrm{~B}$ ). The same experiment was performed using the normal lung fibroblast line, MRC9 (Figure 2B). NMS-P715 alone or in combination with irradiation had no effect on MRC9 survival and radiosensitization. These results suggest that MPS1 is required for cell survival following irradiation of GBM cells but is not required for the survival of normal cells.

\section{Effect of MPS1 inhibition on repair of irradiation induced DNA double-strand breaks}

To assess the effects of NMS-P715 treatment on DNA damage and repair, we analyzed irradiation induced double strand breaks (DSBs) by $\gamma \mathrm{H} 2 \mathrm{AX}$ foci formation (Figure $3 \mathrm{~A}$ and B). At 0.5 and 6 hours, cells treated with radiation or the combination of NMS-P715 and radiation had similar significant elevations in $\gamma \mathrm{H} 2 \mathrm{AX}$ levels compared to control or drug treated cells. At 24 hours, $\gamma \mathrm{H} 2 \mathrm{AX}$ level had returned to near baseline in the cells treated with radiation alone; however, $\gamma \mathrm{H} 2 \mathrm{AX}$ levels were significantly higher in the cells treated with combination NMS-P715 and radiation. On the contrary, normal astrocytes treated with NMS-P715 showed similar number of $\gamma \mathrm{H} 2 \mathrm{AX}$ foci at 24 hour as untreated astrocytes. Moreover, irradiated LN18 and U251 cells treated with $1 \mu$ M NMS-P715 showed statistically more $\gamma \mathrm{H} 2 \mathrm{AX}$ foci $(\mathrm{p}=0.005)$, indicating persistence of radiation induced DNA damaged lesions and enhancement of radiation sensitivity in GBM cells but not in normal cells (Figure 3A). We also observed some induction of $\gamma \mathrm{H} 2 \mathrm{AX}$ foci by NMS-P715 treatment alone. We attribute the DNA damage caused by NMS-P715 to the segregation defects caused by MPS1 inhibition. Representative images of U251 GBM cells and normal astrocytes at 24 hour time point depicting $\gamma \mathrm{H} 2 \mathrm{AX}$ foci - cells stained with anti- $\gamma \mathrm{H} 2 \mathrm{AX}$ antibody followed by Alexa Fluor 488-labeled secondary antibody (Invitrogen) (green) and nuclei with DAPI (blue) are shown (Figure 3B). Given the role of MPS1 as a mitotic kinase, next we looked at mitotic catastrophe as a mechanism of cell death. In addition, LN18 and U251 cells when treated with NMS-P715 and 4-Gy irradiation, showed significant increases in post-irradiation mitotic catastrophe at 48 hours compared to NMS-P715 treated cells $(\mathrm{p}=0.0004$ and 0.002 respectively, Figure $3 \mathrm{C}$ and $\mathrm{D}$ ). As shown in the representative photomicrograph (Figure 3D), U251 cells undergoing mitotic catastrophe are visualized by staining with anti-tubulin antibody (red) and nuclei with DAPI (blue). These results indicate inhibition of MPS1 can increase radiosensitivity of GBM cells by enhancing the number of cells undergoing mitotic catastrophe. The increase in radiosensitivity following MPS1 inhibition could involve the abrogation of double strand break (DSB) repair and increase in 
the number of cells undergoing mitotic catastrophe, contributing to decrease in clonogenic survival.

\section{Molecular profiling reveals changes in genes associated with DNA replication, recombination and repair}

To characterize the molecular mechanism underlying the enhanced radiosensitivity exhibited by GBM cells after MPS1 inhibition, we used gene expression profiling of MPS1 silenced U251 cells. Microarray analysis was used to compare the mRNA expression profile of siMPS1 silenced U251 cells compared to siRNA control (siNeg) transfected cells at 6, 24 and 48 hours post-transfection (Gene Expression Omnibus accession number GSE57091). Two-way hierarchical clustering of mRNA expression profile of siMPS1 silenced U251 cells compared to siNeg transfected or untransfected cells (Control) at 6,24 and 48 hours post-transfection is shown (Supplementary Figure 2A). Values were derived using a cutoff $p$ value $\leq 0.05$ and fold change of $\geq 1.33$. We observed 237, 391 and 891 genes as differentially expressed in siMPS1 silenced U251 cells compared to siNeg-transfected cells at 6,24 and 48 hours respectively. Computational analysis of these differentially expressed genes identified enrichment for genes associated with the Neurological disease, Nervous system development, DNA replication, recombination and repair pathway (DRRRp) (Supplementary Figure 2B \& C). DRRRp was one of the top identified pathways (IPA score, 29), as well as the top molecular and cellular function with 20 molecules affected by downregulation of MPS1 (Supplementary Figure 2B \& C). Genes identified as deregulated following MPS1 loss-of-function that are associated with DNA damage and repair were $D N A P K$ (also known as protein kinase DNA-activated [PRKDC]), and topoisomerase II alpha (TOPO2A).

\section{MPS1 modulates DNA repair molecules in vitro and in vivo}

We confirmed that silencing of MPS1 induces a reduction in DNAPK and TOPO2A mRNA (Supplementary Figure 2D) and protein levels (Figure 4A) in U251 GBM cells and that NMS-P715 also induced a dose dependent decrease in DNAPK and TOPO2A protein in treated U251 cells (Figure 4A). Both siRNA-mediated silencing of MPS1 and small molecule inhibition of MPS1 activity reduced total and phosphorylated DNAPK levels, as well as TOPO2A protein levels (Figure 4A). MPS1 inhibition also downregulated irradiation induced phosphorylated and total DNAPK levels in U251 cells (Supplementary Figure 3A). To determine whether inhibition of MPS1 can modulate DNA repair molecules in vivo, U251 cells grown as xenografts were treated with NMS-P715 $(100 \mathrm{mg} / \mathrm{kg})$ by oral gavage. Twenty-four hours later, tumors were collected and subjected to immunoblot analysis. As shown in Figure (Figure 4 B and C), NMS-P715 treated tumors showed statistically significant reduction in DNAPK ( $\mathrm{p}=0.002)$ and TOPO2A $(\mathrm{p}=0.0003)$ levels, compared to vehicle treated tumors (Figure $4 \mathrm{~B}$ and $\mathrm{C}$ ).

\section{MPS1 regulates NHEJ and HR DNA repair pathways}

Given the altered expression in key members of the DNA damage response - DNAPK and TOPO2A following MPS1 loss of function, we next investigated whether MPSI affects DNA repair efficacy, by examining two major DNA double strand break repair pathways; 
NHEJ and HR (Figure 4 D \& E, Supplementary Figure 3, 4, 5). To assay NHEJ DNA repair pathway, EcoRI endonuclease digested and gel purified plasmid substrate was incubated with nuclear extracts derived from either siNeg or siMPS1 treated U251 cells (Figure 4Di) or NMS-P715 treated U251 cells (Figure 4Dii). Nuclear extracts with functional NHEJ activity can end-join linearized plasmid to form dimers and multimers visualized by agarose gel electrophoresis. In the absence of nuclear extract a single band of the 3.8 kilo base $(\mathrm{kb})$ plasmid is detected (Figure $4 \mathrm{Di}$ and Dii, lane 1). Nuclear extracts from siNeg-transfected U251 cells with intact NHEJ activity showed presence of ligated dimers and multimers (Figure 4 Di, lane 2), whereas nuclear extracts from siMPS1-transfected U251 cells inhibited formation of ligated products, indicating impaired NHEJ DNA repair activity (Figure 4 Di, lane 3). Similarly nuclear extracts from cells with no NMS-P715 treatment showed higher order ligated products, whereas nuclear extracts from cells treated with NMS-P715 inhibited formation of higher order ligated products over time (Figure 4Dii, lanes 3 - 5 vs. lane 2). However, under similar NMS-P715 treatment conditions, U251 cells showed minimal mitotic catastrophe (Supplementary Figure 3C).

Next we analyzed NHEJ activity in the total extracts prepared from U251 xenografts treated with NMS-P715 (Figure 4Diii). In the absence of tumor extract a single band of the 3.8 kilo base (kb) plasmid is detected (Figure 4 Diii, lane 1). However, tumor extracts from vehicle treated U251 tumors showed presence of ligated dimers (Figure 4 Diii, lanes 2-4), whereas tumor extracts from NMS-P715 treated tumors inhibited formation of ligated dimers, indicating impaired NHEJ DNA repair activity (Figure 4 Diii, lanes 5-9). These observations indicate that intact or active MPS1 is necessary for a functional NHEJ pathway in vitro and in vivo in GBM. To assay HR pathway we used a rapid and sensitive PCR based method. The quality control data for the HR assay is shown in the Supplementary Figure 3B. U251 cells were first transfected with siMPS1/siNegative, followed by a second cotransfection with the two HR-plasmids (dl-1 and dl-2) 24 hours after the first transfection. Plasmid DNA was extracted 24 hour after the second transfection and HR activity was quantified using qPCR according to the manufacturer's instructions. The supplied negative and positive primer pairs amplified the expected size of amplicon of similar intensity (Supplementary Figure 3B). As shown in Figure 4E, plasmid DNA extracted from U251 cells treated with different treatments generated a plasmid recombination amplicon of 420 base pairs (bps) as a result of HR activity. The intensity of amplicon is directly correlated to the efficiency of HR activity in the template DNA. The intensity of PCR product generated from siMPS1-transfected cells was lower compared to siNeg-transfected cells (Figure 4E,

lane 3 vs. lane 2). A similar decrease was observed in NMS-P715 treated cells compared to DMSO treated cells (Figure 4E, lane 4 vs. lane 1), indicating MPS1 inhibition mediated decrease in HR activity.

We also examined HR and NHEJ assay using DR-GFP plasmid as given in the supplementary methods (Supplementary Figure 4,5). The HR recombination assay relies on the two inactivated tandem repeated (DR)-GFP transfected cells to express GFP, if HR activity is functional. The results in (Supplementary Figure 4 A-D) clearly show significant $(\mathrm{p}<0.05)$ decrease in percentage GFP+ve cells with MPS1 inhibition both by using siMPS1 and NMS-P715, compared to untreated cells. A RAD51 inhibitor B02 was used as a positive 
control. A decrease in GFP+ve cells indicates inhibition of HR activity. This decrease in HR activity was further confirmed using HR specific primers to amplify the recombined region. As shown, we observed a significant $(\mathrm{p}<0.05)$ decrease in amplification of recombinant PCR product in cells with MPS1 inhibition (Supplementary Figure 4E). A RAD51 inhibitor B02 was used as a positive control. The PCR amplification results are quantified (Supplementary Figure 4F).

Using this well established HR repair system we also verified NHEJ activity in these cells by a PCR based method as described in supplementary materials and methods. As shown in the figure, the enzyme-resistant $650 \mathrm{bp}$ PCR fragment represents functional NHEJ activity Supplementary Figure 5). MPS1 inhibition by both siMPS1 and NMS-P715 treatment, showed lower intensity of enzyme resistant $650 \mathrm{bp}$ product compared to control transfected cells. A DNAPK inhibitor, Nu7441 was used as a positive control for NHEJ repair activity showing similar decrease in enzyme resistant 650 bp PCR product. The differences were statistically significant $(\mathrm{p}<0.05)$ (Supplementary Figure 5A). The PCR amplification results are quantified (Supplementary Figure 5B).

These results indicate MPS1 inhibition can alter HR as well as NHEJ driven DNA repair activity in GBM cells. This further confirms our observations that inhibition of MPS1 leads to the persistence of DNA lesions normally repaired by HR/NHEJ repair pathways.

\section{MPS1 abrogation inhibits GBM tumor growth in vivo}

We next determined if the enhancement of tumor cell radiosensitivity could also extend to an in vivo model using U251 GBM xenografts (Figure 5). To ensure the right dosing schedule, mice bearing U251 GBM xenografts were treated with multiple doses (1-5) of NMS-P715 at $100 \mathrm{mg} / \mathrm{kg}$ concentration and tumors were examined for pDNAPK and total DNAPK levels by an immunoblot analysis (Figure 5A). We observed decrease phosphorylation of DNAPK with all the three tested doses of NMS-P715 ( 1 dose, 3 doses and 5 doses), however administration of 5 doses of NMS-P715 was significantly better in targeting pDNAPK. We also observed downregulation of total DNAPK in treated tumors.

Next, to check the efficacy of MPS1 target in vivo, mice bearing U251 GBM tumors ( 140 $\mathrm{mm}^{3}$ ) were randomized into four groups; vehicle treated controls, NMS-P715 $100 \mathrm{mg} / \mathrm{kg}$ (18), fractionated irradiation (RT), and NMS-P715 plus irradiation (NMS-P715+RT). Based on our dosing schedule data (Figure 5A) in the drug cohort, NMS-P715 was delivered (oral gavage) for 5 doses twice with a two-day gap after the first five doses (total 10 doses). Local irradiation of 4-Gy was given two hours after the fifth and the tenth dose of NMS-P715 to the NMS-P715+RT group and to the RT only group. The growth rates of U251 tumors in different groups are shown (Figure 5B). For each group, the time to grow from $140 \mathrm{~mm}^{3}$ (volume at time of treatment initiation) to $21600 \mathrm{~mm}^{3}$ was calculated using the tumor volumes from the individual mice in each group (mean \pm SEM). The tumor growth rates were similar for U251 tumors treated either with vehicle or NMS-P715 and by day 20, all the mice in control, and NMS-P715 groups had reached the tumor volume of $1600 \mathrm{~mm}^{3}$ and were euthanized. The other two groups, RT and combination (NMS-P715+RT) group were followed until day 33, when at least one mouse in each group reached the tumor volume of $1600 \mathrm{~mm}^{3}$. The absolute growth delays were calculated when mice reached $\geq 600 \mathrm{~mm}^{3}$ 
tumor volume. Vehicle treated control group took 12.4 days ( \pm 0.5 days), drug treated 13.4 days ( \pm 0.5 days ), RT group 26.4 days ( \pm 4 days) and NMS-P715+RT group 32.4 days ( \pm 3 days). At this tumor volume, the growth delay for the NMS-P715 alone and radiation alone groups were 1 day and 14 days, respectively. The growth delay in mice treated with the combination of NMS-P715 and radiation was 20 days, which is greater than the sum of the growth delays caused by NMS-P715 alone and radiation alone. Thus, NMS-P715 delivered alone was not sufficient to have an effect on U251 tumor growth; however in combination with irradiation, the MPS1 inhibitor enhanced radiation-induced tumor growth delay.

\section{MPS1 expression is associated with decreased survival in GBM and other cancer histologies}

We evaluated the prognostic effect of MPSI expression on overall patient survival using a Cox Proportional Hazard model and found lower MPSI expression to be a significant marker of better prognosis in GBM (197 patients), breast (1115 patients) and lung (1405 patients) cancers (Figure 6 and Supplementary Figure 6). Patients with neural GBM classification showed the widest range of MPS1 expression (Figure 6B). High MPS1 expression was associated with a hazard ratio of 2.80 and 1.56 in neural and proneural GBM subtypes respectively. On the contrary, low MPS1 expression is associated with better survival in both GBM subtypes. However, only the neural subtype reached the statistical significance (p-value 0.03) (Table 1). Differences in median survival were also observed when patients were stratified using only MPS1 expression status, with prolonged overall survival of 4.1 months in patients with neural GBM (476 days in low expressers versus 354 days in high expressers) (Figure 6B). However, exclusion of age and MGMT promoter methylation status, two important prognostic factors in GBM, resulted in statistically insignificant p-values. Next, we compared whether the same trend is followed in other cancer types. Univariate survival analysis was performed using data derived from individuals with breast or lung cancer. Kaplan-Meier curve analysis showed patients with MPS1 expression above the median had significantly poorer survival compared to those with MPS1 expression below the median, both in breast cancer $\left(\mathrm{p}\right.$ value $\left.=2 \mathrm{E}^{-04}\right)$ and lung cancer $\left(\mathrm{p}\right.$ value $\left.=1.5 \mathrm{E}^{-08}\right)($ Supplementary Figure 6$)$. The survival analysis clearly shows individuals with tumors expressing low levels of MPS1 had a significant survival advantage when compared to individuals with tumors expressing high levels of MPSI. This confirms MPS1 expression as a potential prognostic marker and as a putative molecular target for cancer therapy.

\section{Discussion}

This study demonstrates that inhibition of MPS1 enhances radiosensitivity of human GBM cells both in vitro and in vivo. The potential for MPS1 inhibition as an effective strategy for cancer treatment has been suggested previously $(11,13)$. However, this is the first study to successfully demonstrate that targeting MPS1 causes radiation sensitization in GBM cells and sheds light on the molecular mechanism of how inhibition of MPS1 activity modulates the growth of cancer cells. The MPS1 kinase has multiple roles in mitosis; these include spindle pole assembly, mitotic exit, cytokinesis and response to genotoxic stress. This implies that MPS1 is near the top of the checkpoint-signaling pathway (8). Upregulation of 
MPS1 has been correlated with increase aneuploidy, higher histological grade and lower survival in human cancers including $\operatorname{GBM}(13,25,26)$.

In this study we show that MPS1 mediates regulation of key DNA repair and damage genes including PRKDC (DNAPK) and TOPO2A. TOPO2A, is highly conserved among eukaryotes and is absolutely required for chromosomal segregation and cell viability (27). Decreased expression of TOPO2A in GBM cells following MPS1 inhibition could be attributed to segregation errors, leading to DNA damage and decreased DNA repair efficacy. Inhibition of MPS1 down-regulated both phosphorylated and total levels of DNAPK in vitro and in vivo. DNAPK is essential for the repair of DNA double-strand breaks (DSB) and has been implicated in - NHEJ and HR repair pathways. Thus, DNAPK is necessary for cellular resistance to ionizing radiation (28). DNAPK plays an instrumental role in many cell processes including cell cycle control, regulation of mitosis, microtubule dynamics, and proper chromosomal segregation. Majority of these cell functions are also regulated by MPS1 (29), it was therefore not surprising to see MPS1 mediated DNAPK regulation in our study.

The direct consequence of MPS1-mediated DNAPK down-regulation was inhibition of NHEJ and HR DNA repair pathways. Eukaryotic cells rely on two highly regulated DSB repair pathways: the NHEJ and HR. Although both NHEJ and HR contribute to DSB rejoining, their involvement varies during the different cell cycle phases as NHEJ is active throughout the cell cycle while HR is active during the S and G2 phases when sister chromatids are available (30). The DNAPK catalytic subunit and Ku heterodimer form the biologically critical DNA-PK complex that plays a crucial role in the repair of ionizing radiation-induced DNA double-strand breaks through NHEJ pathway (31). DNA-PK phosphorylation/autophosphorylation facilitates NHEJ (32). Phosphorylation of DNA-PKcs is complex and occurs at probably more than 40 sites with distinct phosphorylation events result in distinct functional consequences $(33,34)$. The observed NHEJ deficits are likely the consequence of decreased DNAPK levels. Although the role of DNAPK in NHEJ is clear, its role in regulation of HR has not been elucidated. Nonetheless, a recent study showed that NHEJ and HR pathways cooperate in G2 phase cells in order to repair DSB and the impairment of NHEJ by DNAPK inhibitor affects RAD51 recruitment by HR to DSB sites (30). Therefore, our observation of decrease HR activity could be a direct consequence of decreased expression of DNAPK by MPS1 inhibition, as these cells get arrested in G2 phase of cell cycle upon MPS1 abrogation (18).

The reduced DNA repair efficiency in MPS1 inhibited GBM cells was associated with increased retention of $\gamma-\mathrm{H} 2 \mathrm{AX}$ foci and induction of mitotic catastrophe. This effect was enhanced in irradiated cells. Inhibition of MSP1 decreased clonogenic survival ability of GBM cells in conjunction with irradiation. These effects were specific to GBM tumor cells, as normal cells treated with NMS-P715 did not show inhibition of either cell proliferation or decrease in DNA repair and survival potential. Similarly, Slee and colleagues showed that pancreatic ductal adenocarcinoma cells are more sensitive to NMS-P715 inhibition than the adipose-derived normal mesenchymal stem cells (26). Consequently, the differences in sensitivity between tumor and normal cells could provide a favorable therapeutic window in the treatment of GBM. 
To further evaluate the clinical potential of NMS-P715 delivered in combination with radiotherapy we evaluated this treatment strategy in a preclinical model system. U251 tumor xenografts treated with NMS-P715 showed significant increase in radiation-induced tumor growth delay. This coincided with the down-regulation of DNAPK in tumor xenografts. This is important as DNAPK is required to prevent mitotic catastrophe in response to DNA damage and is associated with radioresistance in glioma $(28,35)$.

Our model for sensitivity to MPS1 inhibition depends on NHEJ and HR deficiency in MPS1 depleted cells. Therefore, this approach may be more widely applicable in the treatment of variety multitude of cancers. In order to test this, we looked at MPSI expression and patient survival in GBM patient samples from the TCGA database stratified according to their GBM molecular subtypes (classical, mesenchymal, proneural, and neural) (36) and two other tumor histologies, breast cancer and lung cancer. Using a Cox Proportional Hazards model, we observed that lower MPS1 expression to be a significant marker of better prognosis in GBMs of neural classification, and breast and lung cancers. Similar findings have been reported recently where it was shown that MPS1 inhibition enhances chemosensitivity of GBM to anti-mitotic drugs (25). Together, these findings suggest that MPS1 is a promising molecular target in the treatment of GBM. Additionally, MPS1 has prognostic value not only in a subtype of GBM patients, but also in patients with other cancer histologies. Many of the conventional approaches to the treatment of advanced cancers are genotoxic and therefore trigger a DNA damage response leading to cell cycle checkpoint response (37). Intact checkpoint responses can reduce sensitivity to these agents $(38,39)$. The reduced repairability of DNA damage using specific repair pathways in MPS1 deficient tumor cells could be exploitable in combination with radiotherapy for the treatment of cancer.

\section{Supplementary Material}

Refer to Web version on PubMed Central for supplementary material.

\section{Acknowledgements}

This research was supported by the Intramural Research Program of the National Institutes of Health, National Cancer Institute.

\section{References}

1. Draviam VM, Xie S, Sorger PK. Chromosome segregation and genomic stability. Current opinion in genetics \& development. 2004; 14:120-125. [PubMed: 15196457]

2. Weaver BA, Cleveland DW. Decoding the links between mitosis, cancer, and chemotherapy: The mitotic checkpoint, adaptation, and cell death. Cancer cell. 2005; 8:7-12. [PubMed: 16023594]

3. Suijkerbuijk SJ, Kops GJ. Preventing aneuploidy: the contribution of mitotic checkpoint proteins. Biochimica et biophysica acta. 2008; 1786:24-31. [PubMed: 18472014]

4. Gordon DJ, Resio B, Pellman D. Causes and consequences of aneuploidy in cancer. Nature reviews Genetics. 2012; 13:189-203.

5. Singh D, Chan JM, Zoppoli P, Niola F, Sullivan R, Castano A, et al. Transforming fusions of FGFR and TACC genes in human glioblastoma. Science. 2012; 337:1231-1235. [PubMed: 22837387]

6. Mills GB, Schmandt R, McGill M, Amendola A, Hill M, Jacobs K, et al. Expression of TTK, a novel human protein kinase, is associated with cell proliferation. The Journal of biological chemistry. 1992; 267:16000-16006. [PubMed: 1639825] 
7. Sun T, Yang X, Wang W, Zhang X, Xu Q, Zhu S, et al. Cellular abundance of Mps1 and the role of its carboxyl terminal tail in substrate recruitment. The Journal of biological chemistry. 2010; 285:38730-38739. [PubMed: 20884615]

8. Liu X, Winey M. The MPS1 family of protein kinases. Annual review of biochemistry. 2012; 81:561-585.

9. Kasbek C, Yang CH, Fisk HA. Mps1 as a link between centrosomes and genomic instability. Environmental and molecular mutagenesis. 2009; 50:654-665. [PubMed: 19274768]

10. Fisk HA, Mattison CP, Winey M. A field guide to the Mps1 family of protein kinases. Cell Cycle. 2004; 3:439-442. [PubMed: 14963409]

11. Yuan B, Xu Y, Woo JH, Wang Y, Bae YK, Yoon DS, et al. Increased expression of mitotic checkpoint genes in breast cancer cells with chromosomal instability. Clinical cancer research : an official journal of the American Association for Cancer Research. 2006; 12:405-410. [PubMed: 16428479]

12. Kilpinen S, Ojala K, Kallioniemi O. Analysis of kinase gene expression patterns across 5681 human tissue samples reveals functional genomic taxonomy of the kinome. PloS one. 2010; 5:e15068. [PubMed: 21151926]

13. Daniel J, Coulter J, Woo JH, Wilsbach K, Gabrielson E. High levels of the Mps1 checkpoint protein are protective of aneuploidy in breast cancer cells. Proceedings of the National Academy of Sciences of the United States of America. 2011; 108:5384-5389. [PubMed: 21402910]

14. Wei JH, Chou YF, Ou YH, Yeh YH, Tyan SW, Sun TP, et al. TTK/hMps1 participates in the regulation of DNA damage checkpoint response by phosphorylating CHK2 on threonine 68. The Journal of biological chemistry. 2005; 280:7748-7757. [PubMed: 15618221]

15. Yeh YH, Huang YF, Lin TY, Shieh SY. The cell cycle checkpoint kinase CHK2 mediates DNA damage-induced stabilization of TTK/hMps1. Oncogene. 2009; 28:1366-1378. [PubMed: 19151762]

16. Delaney G, Jacob S, Featherstone C, Barton M. The role of radiotherapy in cancer treatment: estimating optimal utilization from a review of evidence-based clinical guidelines. Cancer. 2005; 104:1129-1137. [PubMed: 16080176]

17. Begg AC, Stewart FA, Vens C. Strategies to improve radiotherapy with targeted drugs. Nature reviews Cancer. 2011; 11:239-253.

18. Colombo R, Caldarelli M, Mennecozzi M, Giorgini ML, Sola F, Cappella P, et al. Targeting the mitotic checkpoint for cancer therapy with NMS-P715, an inhibitor of MPS1 kinase. Cancer research. 2010; 70:10255-10264. [PubMed: 21159646]

19. Shankavaram U, Fliedner SM, Elkahloun AG, Barb JJ, Munson PJ, Huynh TT, et al. Genotype and tumor locus determine expression profile of pseudohypoxic pheochromocytomas and paragangliomas. Neoplasia. 2013; 15:435-447. [PubMed: 23555188]

20. Iliakis G, Rosidi B, Wang M, Wang H. Plasmid-based assays for DNA end-joining in vitro. Methods Mol Biol. 2006; 314:123-131. [PubMed: 16673879]

21. Camphausen K, Burgan W, Cerra M, Oswald KA, Trepel JB, Lee MJ, et al. Enhanced radiationinduced cell killing and prolongation of gammaH2AX foci expression by the histone deacetylase inhibitor MS-275. Cancer research. 2004; 64:316-321. [PubMed: 14729640]

22. Comprehensive genomic characterization defines human glioblastoma genes and core pathways. Nature. 2008; 455:1061-1068. [PubMed: 18772890]

23. Tandle AT, Kramp T, Kil WJ, Halthore A, Gehlhaus K, Shankavaram U, et al. Inhibition of pololike kinase 1 in glioblastoma multiforme induces mitotic catastrophe and enhances radiosensitisation. Eur J Cancer. 2013; 49:3020-3028. [PubMed: 23790466]

24. Diggle CP, Bentley J, Kiltie AE. Development of a rapid, small-scale DNA repair assay for use on clinical samples. Nucleic acids research. 2003; 31:e83. [PubMed: 12888535]

25. Tannous BA, Kerami M, Van der Stoop PM, Kwiatkowski N, Wang J, Zhou W, et al. Effects of the selective MPS1 inhibitor MPS1-IN-3 on glioblastoma sensitivity to antimitotic drugs. Journal of the National Cancer Institute. 2013; 105:1322-1331. [PubMed: 23940287]

26. Slee RB, Grimes BR, Bansal R, Gore J, Blackburn C, Brown L, et al. Selective Inhibition of Pancreatic Ductal Adenocarcinoma Cell Growth by the Mitotic MPS1 Kinase Inhibitor NMSP715. Molecular cancer therapeutics. 2014; 13:307-315. [PubMed: 24282275] 
27. Warburton PE, Earnshaw WC. Untangling the role of DNA topoisomerase II in mitotic chromosome structure and function. BioEssays : news and reviews in molecular, cellular and developmental biology. 1997; 19:97-99.

28. Mahaney BL, Meek K, Lees-Miller SP. Repair of ionizing radiation-induced DNA double-strand breaks by non-homologous end-joining. The Biochemical journal. 2009; 417:639-650. [PubMed: 19133841]

29. Lee KJ, Lin YF, Chou HY, Yajima H, Fattah KR, Lee SC, et al. Involvement of DNA-dependent protein kinase in normal cell cycle progression through mitosis. The Journal of biological chemistry. 2011; 286:12796-12802. [PubMed: 21330363]

30. Bee L, Fabris S, Cherubini R, Mognato M, Celotti L. The efficiency of homologous recombination and non-homologous end joining systems in repairing double-strand breaks during cell cycle progression. PloS one. 2013; 8:e69061. [PubMed: 23874869]

31. Dobbs TA, Tainer JA, Lees-Miller SP. A structural model for regulation of NHEJ by DNA-PKcs autophosphorylation. DNA repair. 2010; 9:1307-1314. [PubMed: 21030321]

32. Uematsu N, Weterings E, Yano K, Morotomi-Yano K, Jakob B, Taucher-Scholz G, et al. Autophosphorylation of DNA-PKCS regulates its dynamics at DNA double-strand breaks. The Journal of cell biology. 2007; 177:219-229. [PubMed: 17438073]

33. Meek K, Dang V, Lees-Miller SP. DNA-PK: the means to justify the ends? Advances in immunology. 2008; 99:33-58. [PubMed: 19117531]

34. Neal JA, Dang V, Douglas P, Wold MS, Lees-Miller SP, Meek K. Inhibition of homologous recombination by DNA-dependent protein kinase requires kinase activity, is titratable, and is modulated by autophosphorylation. Molecular and cellular biology. 2011; 31:1719-1733. [PubMed: 21300785]

35. Shang ZF, Huang B, Xu QZ, Zhang SM, Fan R, Liu XD, et al. Inactivation of DNA-dependent protein kinase leads to spindle disruption and mitotic catastrophe with attenuated checkpoint protein 2 Phosphorylation in response to DNA damage. Cancer research. 2010; 70:3657-3666. [PubMed: 20406977]

36. Verhaak RG, Hoadley KA, Purdom E, Wang V, Qi Y, Wilkerson MD, et al. Integrated genomic analysis identifies clinically relevant subtypes of glioblastoma characterized by abnormalities in PDGFRA, IDH1, EGFR, and NF1. Cancer cell. 2010; 17:98-110. [PubMed: 20129251]

37. Pavey S, Spoerri L, Haass NK, Gabrielli B. DNA repair and cell cycle checkpoint defects as drivers and therapeutic targets in melanoma. Pigment cell \& melanoma research. 2013

38. Barckhausen C, Roos WP, Naumann SC, Kaina B. Malignant melanoma cells acquire resistance to DNA interstrand cross-linking chemotherapeutics by p53-triggered upregulation of DDB2/XPCmediated DNA repair. Oncogene. 2013

39. Silver DP, Richardson AL, Eklund AC, Wang ZC, Szallasi Z, Li Q, et al. Efficacy of neoadjuvant Cisplatin in triple-negative breast cancer. Journal of clinical oncology : official journal of the American Society of Clinical Oncology. 2010; 28:1145-1153. [PubMed: 20100965] 
Implications

Implications: Inhibition of MPS1 kinase in combination with radiation represents a promising new approach for glioblastoma and for other cancer therapies. 
A

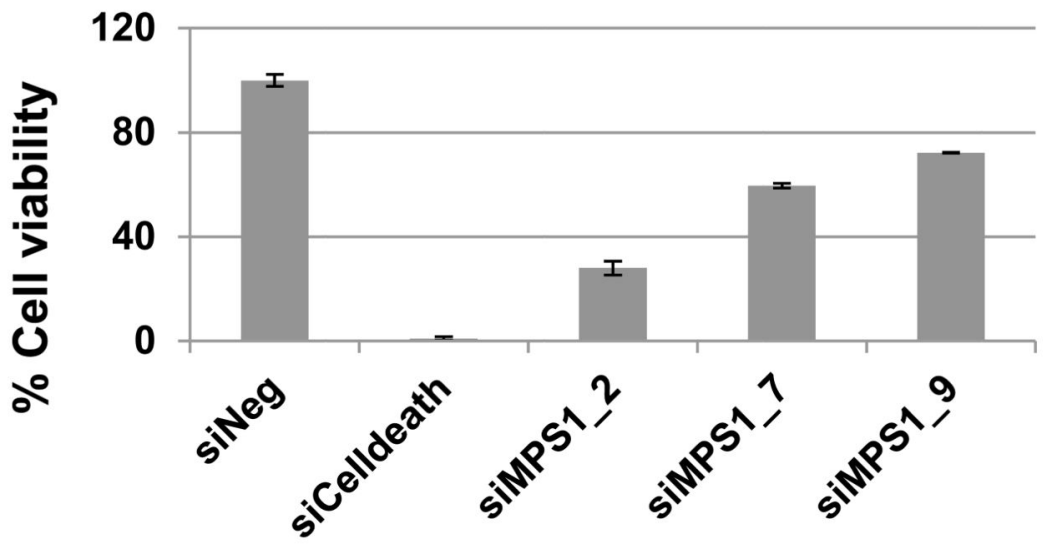

B

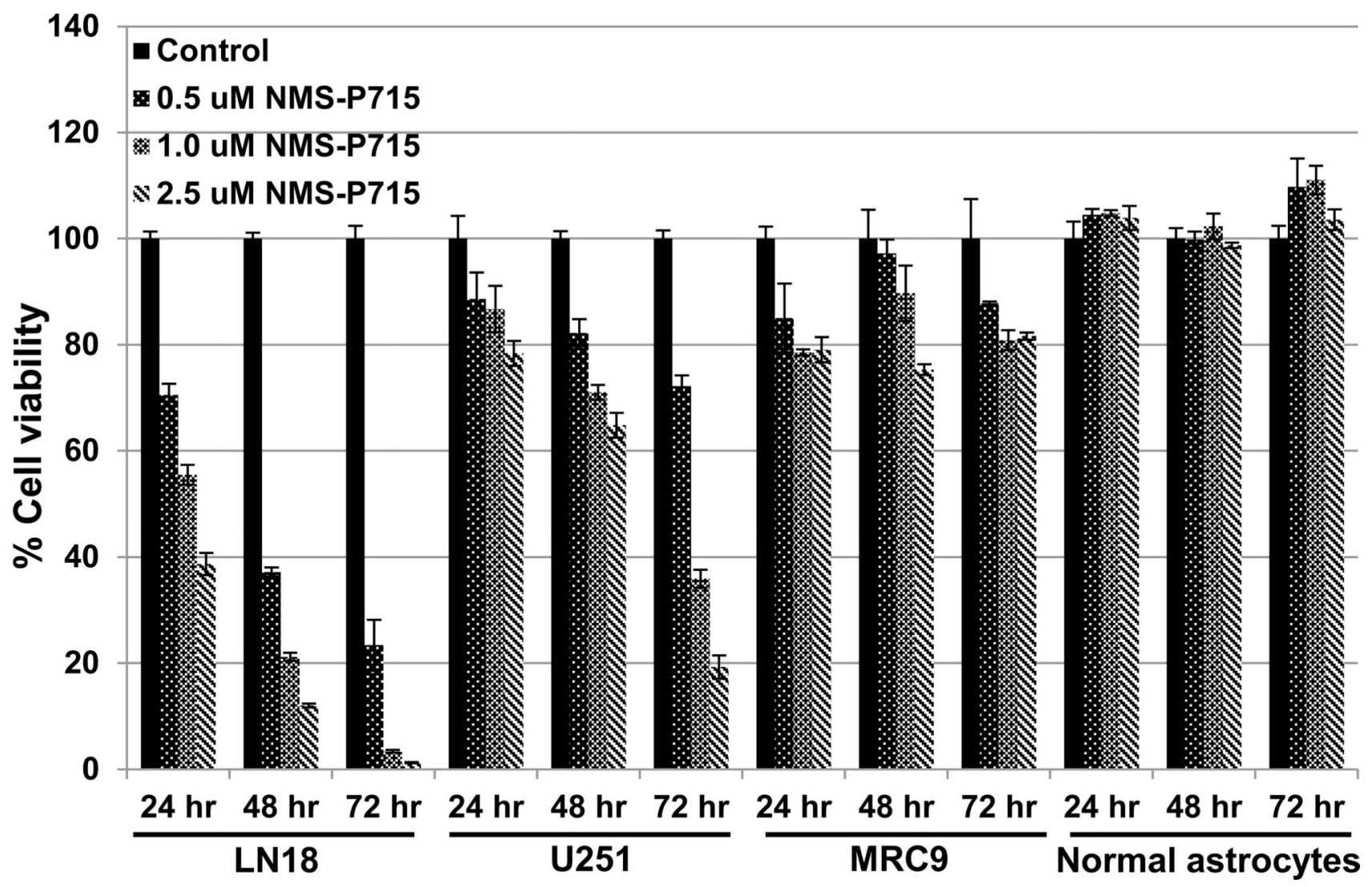

Figure 1. Inhibition of MPS1 activity compromises the viability of GBM tumor cells

The human GBM cell line U251 was transfected with three different non-overlapping siRNAs corresponding to MPS1 (siMSP1), or negative (siNeg) siRNA in triplicate. Cell viability A was used to assess the phenotypic effects at 72 hours post siRNA transfection. siCelldeath was used as a positive control. Data presented are the mean \pm the standard deviation relative to siNeg-transfected cells. B LN18 and U251 (GBM tumor cells) and MRC9 (normal fibroblasts) and normal astrocytes were exposed to NMS-P715 (0.5, 1.0, or $2.5 \mu \mathrm{M}$ ) or DMSO (control). Cell viability (Cell Titer Glo) was assessed 24, 48 and 72 hours and compared to DMSO treated cells. Data is shown as mean \pm standard deviation. 


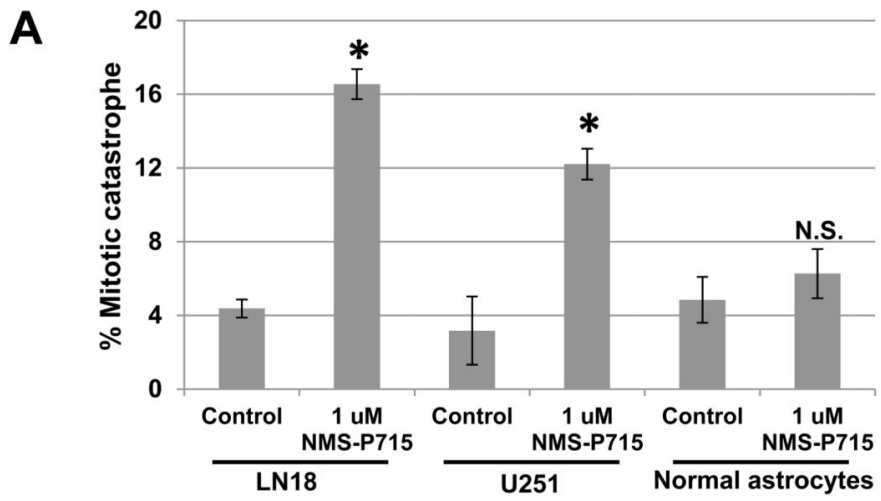

B
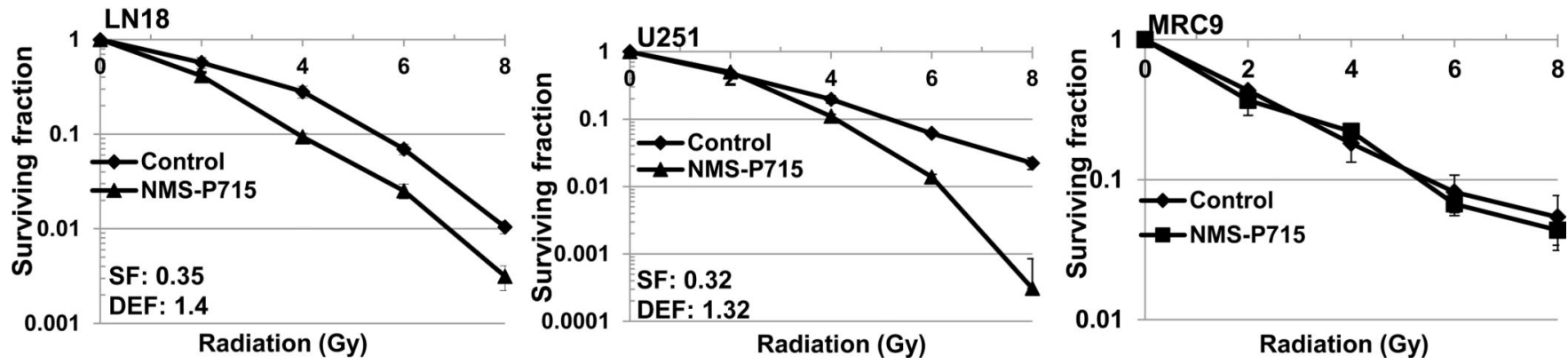

Figure 2. Pharmacologic MPS1 inhibition induced mitotic catastrophe and decreased clonogenic survival in GBM cells

(A) To assess mitotic catastrophe represented as the number of cells with multilobulated giant nuclei and cells with abnormal mitoses, LN18 and U251 cells and normal astrocytes stained with anti-tubulin antibody and nuclei were visualized with DAPI staining. Values represent the mean standard deviation for 3 independent experiments, * indicates $\mathrm{p}<0.05$ according to the Student $t$ test. (B) MPS1 inhibition enhances the radiosensitivity of GBM cells. Cells were exposed to $1 \mu \mathrm{M}$ NMS-P715 or DMSO control and clonogenic survival was assessed. Colony-forming efficiency was determined in GBM tumor cells LN18, U251 and normal fibroblast cell line (MRC9). Ten to 14 days later survival curves were generated after normalizing for the cytotoxicity induced by NMS-P715 alone and surviving fraction (SF) and dose enhancement factor (DEF) were calculated. Values represent the mean standard deviation for 3 independent experiments, * indicates $\mathrm{p}<0.05$ according to the Student $t$ test. 


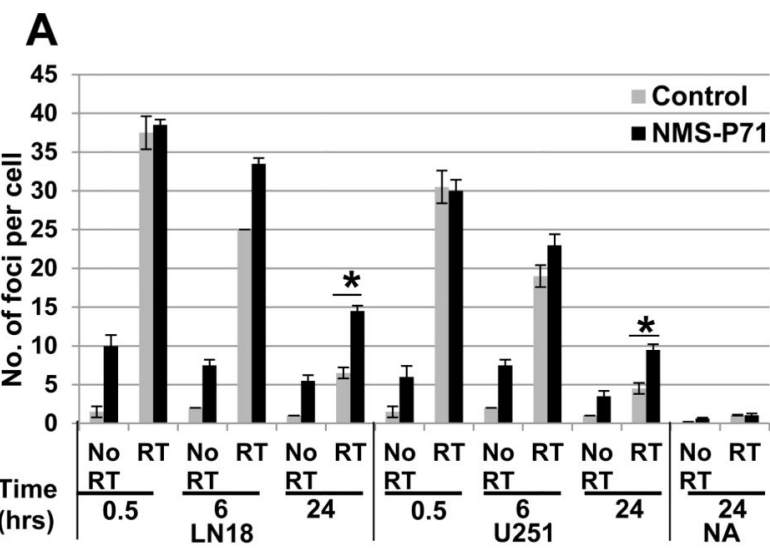

B

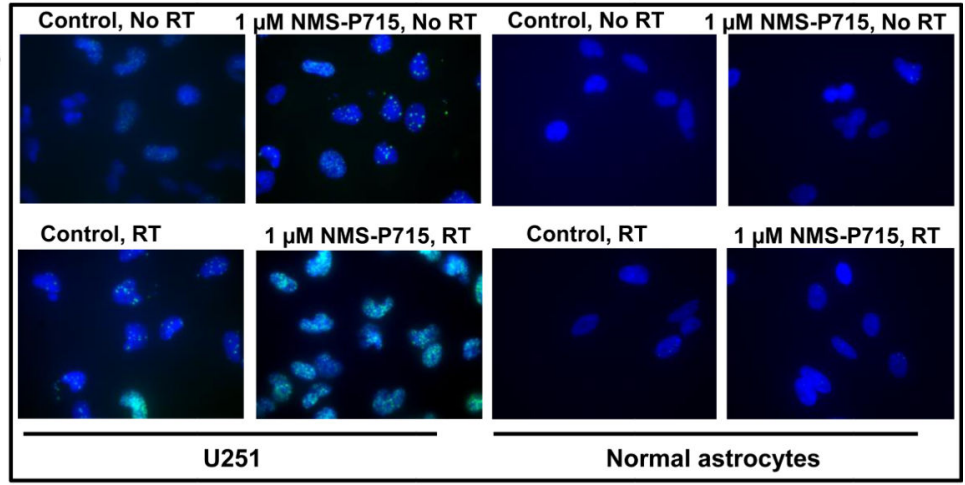

\section{C}

D
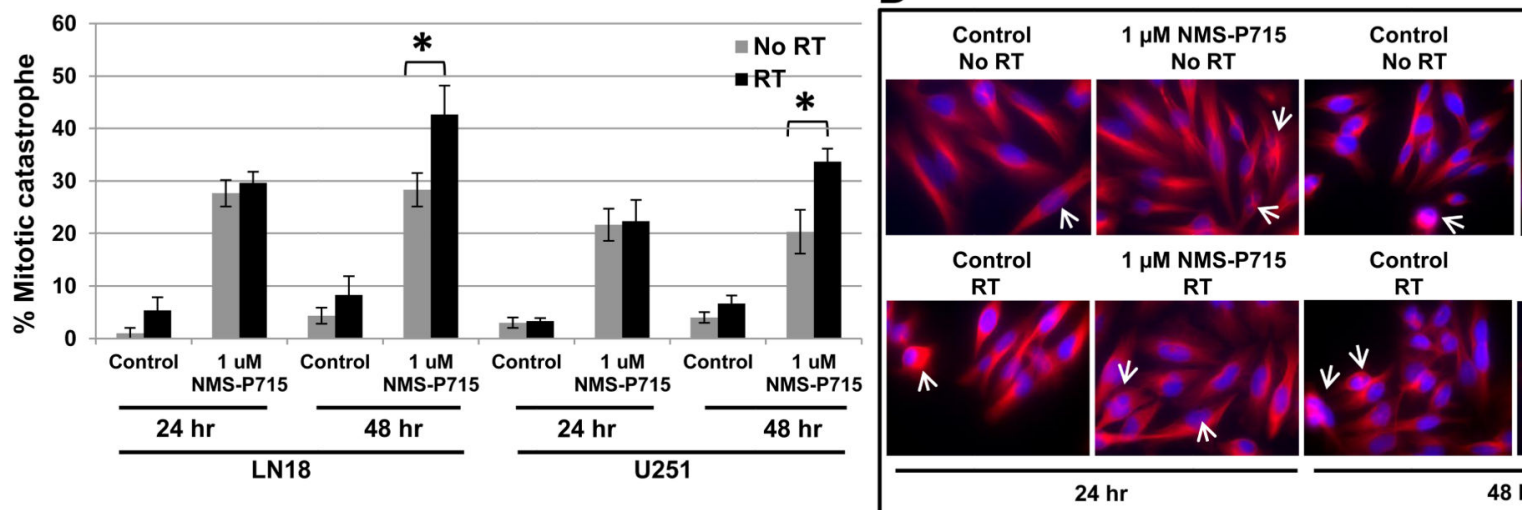
No RT

Figure 3. Effect of MPS1 inhibition on repair of radiation induced DNA double-strand breaks (A,B) Immunocytochemical staining to detect $\gamma \mathrm{H} 2 \mathrm{AX}$ foci was performed. LN18, U251 tumor cells and normal astrocytes were plated in four-well chamber slides, allowed to attach (overnight) and then treated with NMS-P715 for two hours prior to irradiation (4-Gy). (A) Foci were counted at $0.5,6$ and 24 hours postradiation in at 50 cells per experiment. Data presented are the mean \pm SD from at least three independent experiments. $(\mathbf{B})$ Representative images of U251 GBM cells and normal astrocytes showing $\gamma \mathrm{H} 2 \mathrm{AX}$ foci, cells stained with anti- $\gamma \mathrm{H} 2 \mathrm{AX}$ antibody followed by Alexa Fluor 488-labeled secondary antibody (green) and nuclei were visualized with DAPI (blue) staining. (C,D) LN18 and U251 cells growing in chamber slides were exposed to NMS-P715 for two hours, irradiated (4-Gy), and 24 hours and 48 hours after irradiation processed for immunocytochemical analysis of mitotic catastrophe. (C) Nuclear fragmentation (defined as the presence of two or more distinct lobes within a single cell) was evaluated in at least 150 cells per treatment per experiment. * indicates $\mathrm{p}<0.05$ comparing cells in the combination group compared to either drug or radiation alone groups at the same time point. (D) Representative images of U251 GBM cells showing mitotic catastrophe, cells stained with anti-tubulin antibody (red) and nuclei were visualized with DAPI (blue) staining 

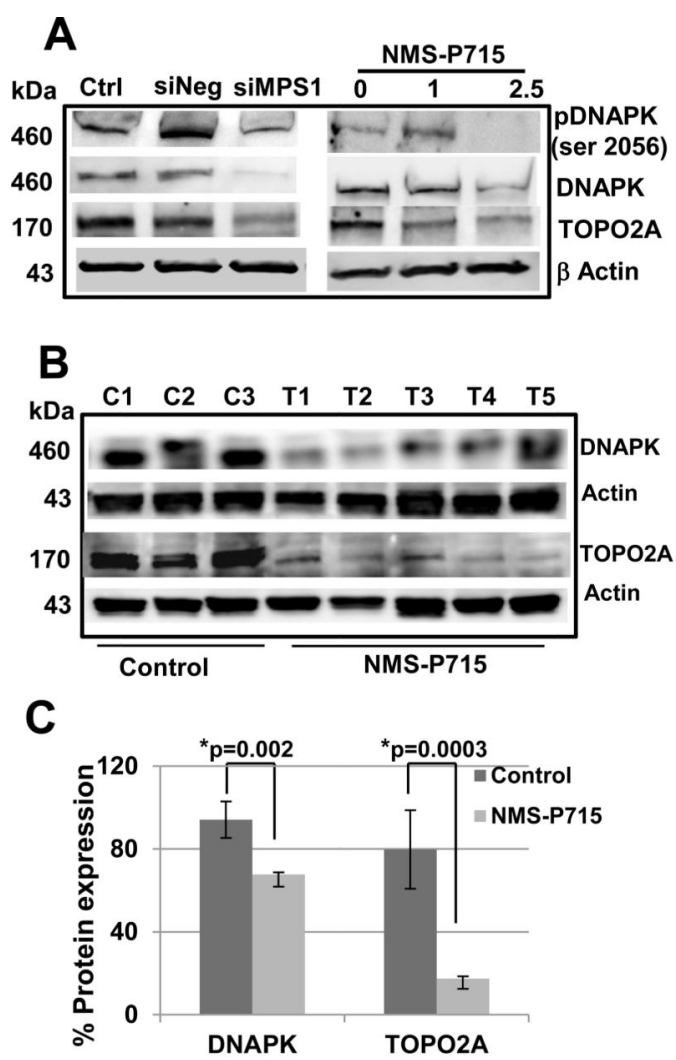

D

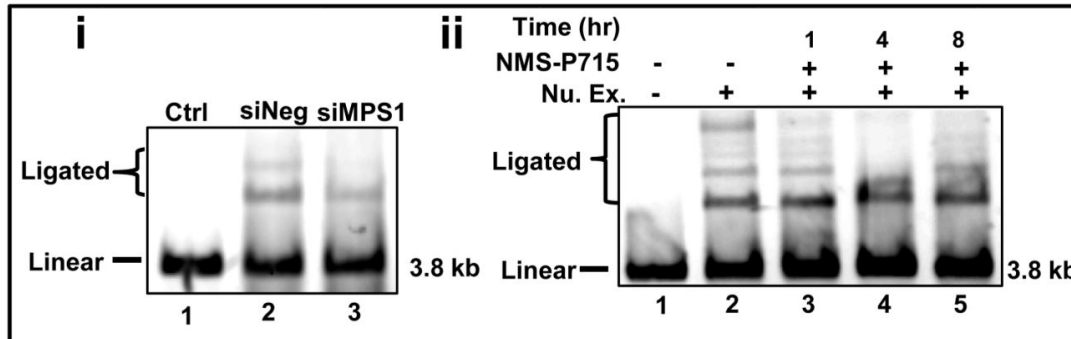

iii

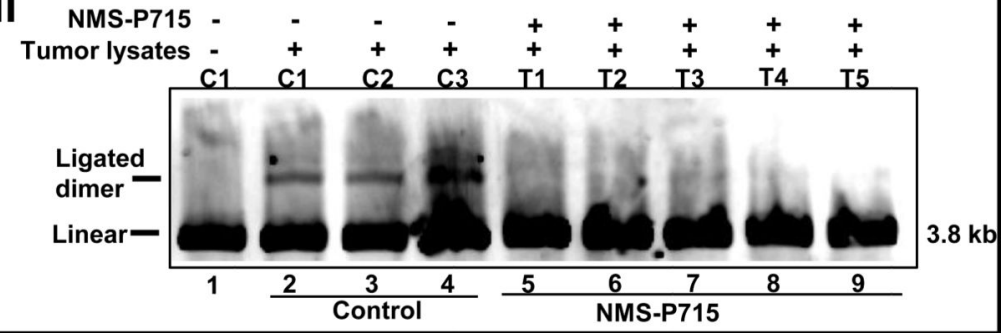

E

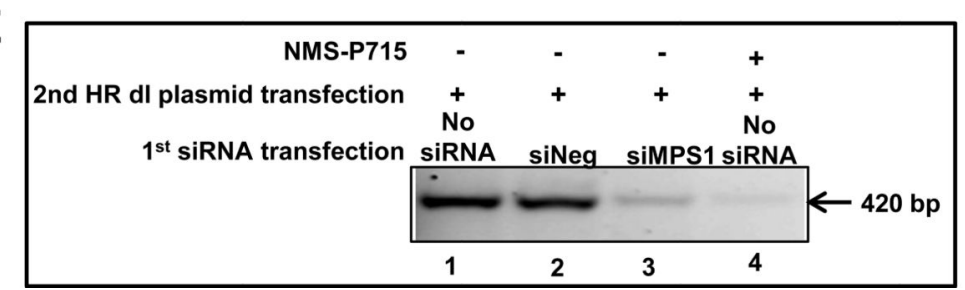

Figure 4. MPS1 modulates DNA repair molecules and DNA repair pathways

(A) Western blot analysis of pDNAPK, DNAPK and TOPO2A DNA repair proteins from siRNA (left) and NMS-P715 (right) treated U251 cells. Actin was used as a loading control. Western blot analysis (B) and quantitation $(\mathbf{C})$ of DNAPK and TOPO2A protein levels from vehicle (Control C1 - C3) and NMS-P715 (T1 - T5) treated U251 tumors. Actin levels were measured as a loading control. (D) To detect non-homologous end joining DNA repair activity, an Eco RI endonuclease digested and gel purified plasmid substrate was incubated with nuclear extracts derived from either siNeg or siMPS1 treated U251 cells (Di) or NMSP715 treated U251 cells (Dii) or total tumor lysates from U251 tumors (vehicle (Control C1 - C3) and NMS-P715 (T1 - T5) treated U251 tumors as in Figure 5B) (Diii). The reaction mixture was electrophoresed on agarose gel to visualize rejoined products by staining with SybrGreen. (E) To assay homologous recombination (HR) activity, U251 cells were first transfected with siMPS1/siNegative (lanes 2 and 3) or treated with DMSO/NMS-P715 (1 $\mu \mathrm{M})$ (lanes 1 and 4), followed by a second co-transfection with the two HR $\mathrm{dl}$ (dl-1 and dl-2) plasmids 24 hours after the first transfection. Plasmid DNA was extracted 24 hours after the second transfection and HR activity was quantified through qPCR using the supplied primers. 


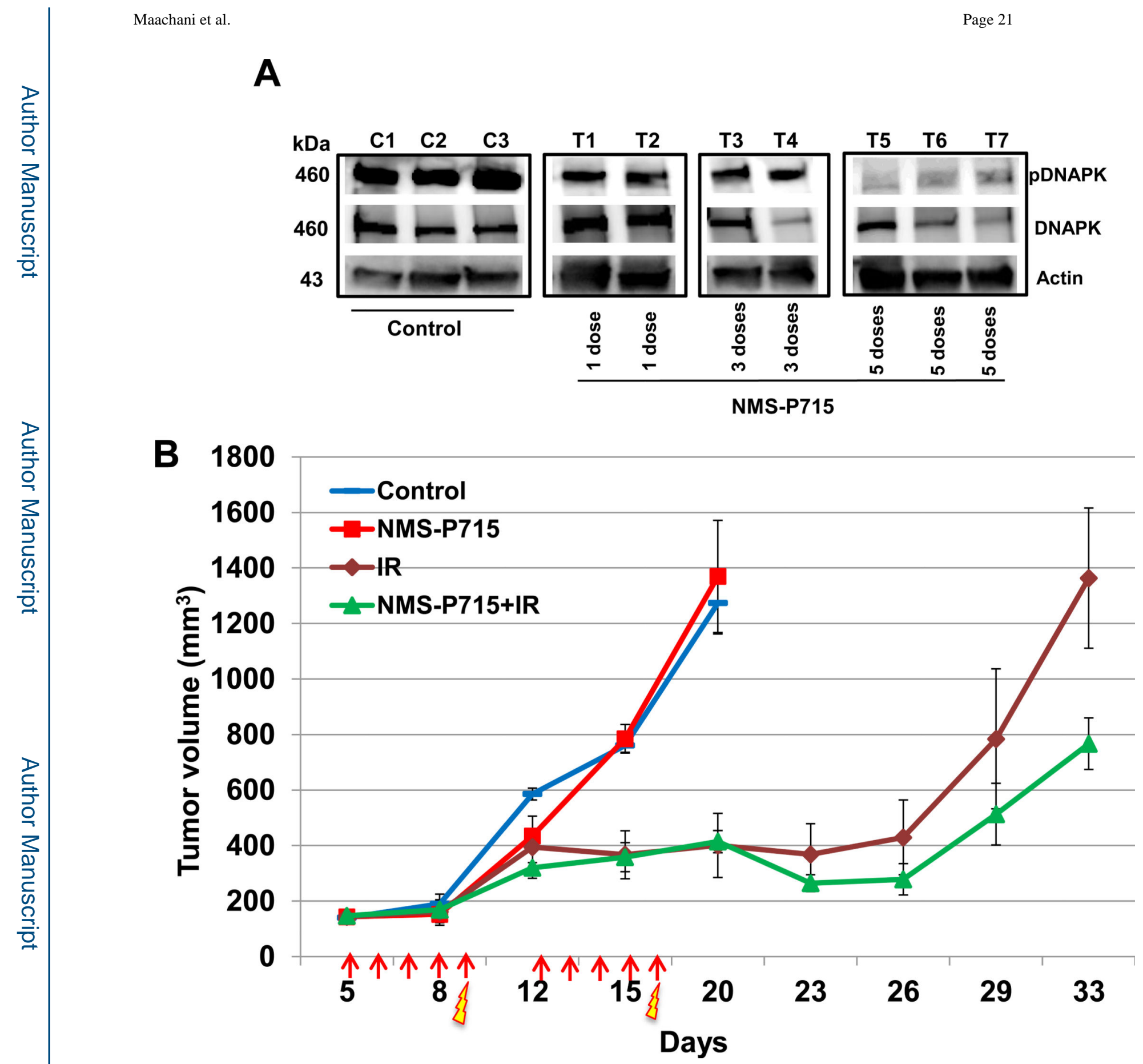

Figure 5. MPS1 abrogation inhibits MPS1 targets and GBM tumor growth in-vivo Mice were implanted with $5 \times 10^{6}$ U251cells on the lateral aspect of the rear leg. (A) Animals were treated with $100 \mathrm{mg} / \mathrm{kg}$ of NMS-P715 either once ( 1 dose), three times ( 3 doses) or 5 times ( 5 doses) by oral gavage. The multiple doses were 24 hours apart. Tumors were examined 6 hours after the last dose by an immunoblot analysis. (B) Tumors were randomized into four groups and treated with either vehicle control, NMS-P715 (100 mg/kg by oral gavage, ten treatments over two weeks), radiotherapy (4-Gy, two treatments over two weeks), and combination treatment with NMS-P715 and radiotherapy. Tumors were 
measured two times per week and followed until they reached at least $2,000 \mathrm{~mm}^{3}$. Volumes were calculated using the formula $\left(L \times W^{2}\right) \times \pi / 2$. 


\section{A}
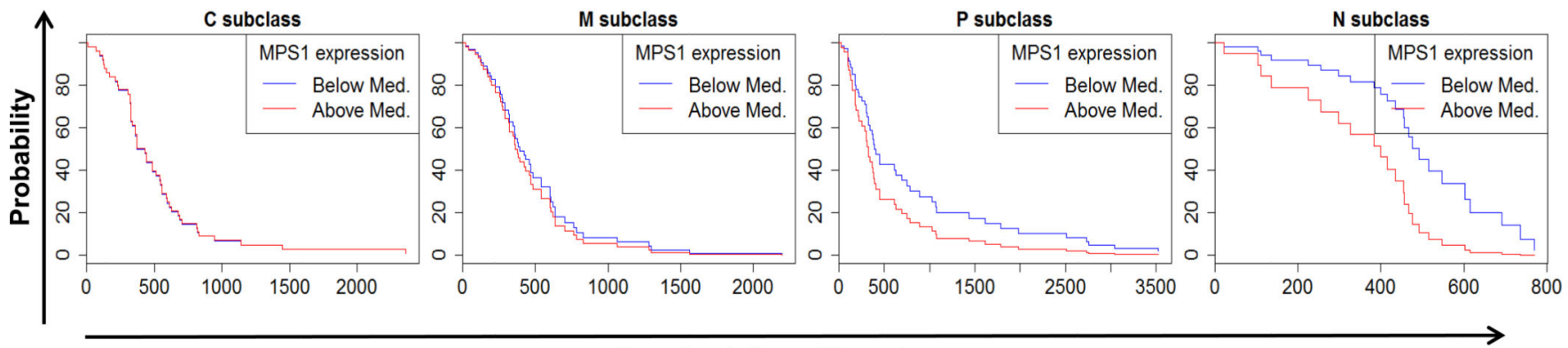

Survival time (Days)

B

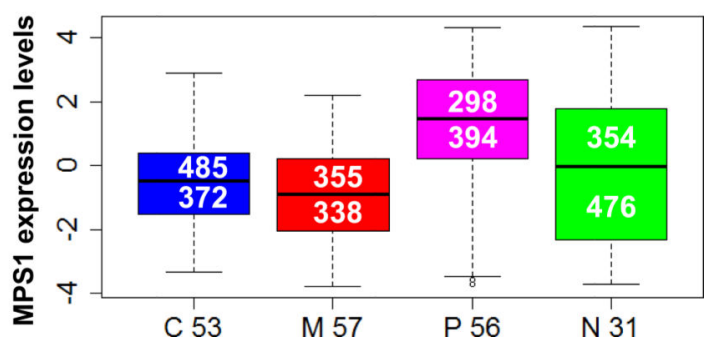

Figure 6.

(A) Multivariate survival analysis was carried out using a Cox Proportional Hazard model on 197 patients from TCGA datasets (https://tcga-data.nci.nih.gov) examining MPS1 expression (red- above median expression, blue- below median expression) and overall survival association in different subclasses (C-classical, M-mesenchymal, $\mathrm{P}$ - proneural and N- neural) of GBM. (B) MPS1 expression and survival period for various GBM subtypes (C-classical, M-mesenchymal, P- proneural and N- neural) from TCGA datasets. The number indicates number of patients in each subtype. 


\section{Table 1}

Multivariate survival analysis for various GBM subtypes

\begin{tabular}{ccccc}
\hline GBM Subclass & $\mathbf{C}$ & $\mathbf{M}$ & $\mathbf{P}$ & $\mathbf{N}$ \\
\hline Logrank p value & 0.382 & 0.009 & 0.004 & 0.029 \\
\hline Exp. HR (p value) & $0.986(0.965)$ & $1.157(0.633)$ & $1.567(0.158)$ & $2.803(0.035)$ \\
\hline
\end{tabular}

C: classical, M: mesenchymal, P: proneural, N: neural 